Williams, M. A. \& Rittenberg, S. C. (1956). J. gen. Microbiol. 15, 205-209

\title{
Microcyst Formation and Germination in Spirillum lunatum
}

\author{
BY MARION A. WILLIAMS \\ Biological Research Laboratory, Southern Illinois University, Carbondale, \\ Illinois, U.S.A. \\ AND S. C. RITTENBERG \\ Department of Bacteriology, University of Southern California, Los Angeles, \\ California, U.S.A.
}

SUMMARY: Spirillum lunatum has been shown to possess a life cycle in which there is an alternation between a vegetative and a microcyst stage. Microcyst formation may occur from the spiral vegetative cell by:(1) fusion of two entwined organisms to form one or more microcysts ; $(2)$ the production of a protuberance at some point along the organism into which the entire organism is gradually absorbed; (3) the gradual shortening and rounding of the organism to form an oval to spherical body. Microcyst formation begins, in a typical broth culture, at $c .24 \mathrm{hr}$. after inoculation and the majority of the organisms are in the microcyst stage after a 4-day incubation period. When microcysts from an old culture are inoculated into fresh media, they germinate to form the spiral vegetative organism. Germination occurs by either unipolar or bipolar emergence of the germ tube.

During a taxonomic study of the genus Spirillum Ehrenberg (Williams, 1952), it was found that three species of the marine spirilla and two of the freshwater species exhibited morphological changes during the growth of the cultures. Young cultures, which consisted entirely of spiral organisms, gradually changed until only oval or spherical bodies $(0 \cdot 8-5 \cdot 0 \mu$. in diameter, depending on the species) were found in old cultures. When these bodies were inoculated into fresh media, the normal spiral organism germinated from them. Since the entire organism is involved in the formation of these oval to spherical bodies, in a manner similar to the formation of the microcysts of the Sporocytophaga (Stanier, 1942; Grace, 1951), these bodies have been called ' microcysts'. Because of its relatively large size, S. lunatum was chosen as the experimental organism. The morphological changes which occur in this species are similar, although not identical, to those occurring in the other two marine spirilla and in the two freshwater species.

\section{METHODS}

The spirillum was grown in sea-water nutrient broth of the following composition: peptone, 5.0 g.; beef extract, 3.0 g.; yeast autolysate, 3.0 g.; and sea water, $1000 \mathrm{ml}$. Broth tubes were inoculated with old cultures, ranging in age from 2 to 6 weeks, and incubated at $30^{\circ}$. Organisms removed from the broth cultures at appropriate times between 8 and $72 \mathrm{hr}$. were used for microcultures. Cultures less than $24 \mathrm{hr}$. old were centrifuged and the harvested organisms re-suspended in a small amount of sterile sea water. Cultures older than $24 \mathrm{hr}$. 
always form a pellicle and suspensions of the older organisms were prepared by emulsifying a loopful of pellicle in $1 \mathrm{ml}$. of sterile sea water.

Microcultures were used to obtain photographs of the changes which occurred in the transformation of the spiral organisms into microcysts and to show the germination of the microcysts into the vegetative spiral organisms. They were prepared by placing a drop of suspension on a sterile glass slide and covering the preparation with a sterile $\mathbf{O}$ coverslip. Excess fluid was removed with blotting-paper and the preparation sealed with immersion oil. The processes of microcyst formation and germination were observed in individual organisms by dark phase-contrast microscopy and photographed serially as the changes occurred. In the serial photographs the letters ' $A$ ', ' $B$ ' ' $C$ ', . ., indicate the order of the sequence. The time, in minutes, is shown in the lower right-hand corner with the beginning of a sequence taken as zero time.

\section{RESULTS}

\section{Microcyst formation}

From entwined and fused organisms. When an old culture of Spirillum lunatum is inoculated into fresh medium, there is little evidence of growth until the culture is approximately $24 \mathrm{hr}$. old. At this time, the culture is found to consist entirely of the spiral organisms considered normal for the genus Spirillum (Pl. 1, fig. 1). These spiral organisms divide by transverse fission during the next 10-12 hr. During this time the majority of the organisms are actively motile; however, a few become motionless. When a freely motile organism comes in the vicinity of a motionless one, the motile one appears to become attracted to, and may become entwined about the motionless organism. During the entwining process, which may last for several hours, the organisms are in violent motion and it is not possible to obtain still photographs of the early stages. Eventually the two entwined organisms become motionless, and when this occurs, it can be seen that the two organisms have fused (Pl. 1, fig. 2; Pl. 2, fig. 3). As time elapses, the entwined organisms become shorter and thicker and a swelling occurs (Pl. 1, fig. $2 \mathrm{E}$ ) or a protuberance forms (Pl. 2, fig. 3C) at the point of fusion, which gradually enlarges, absorbing the rest of the fused organisms, to form the microcysts (Pl. 1, fig. 2; Pl. 2, fig. 3).

In some instances there is only one point of fusion between the two entwined organisms and only a single microcyst is formed, but in the majority of cases more than one microcyst is formed from a pair of fused organisms. The number of microcysts produced appears to be related to the number of fusion points. It is difficult to show both organisms of a fusion pair in their entirety by photographs of a particular focal depth. In the sequence shown in Pl. 2, fig. 3, different points of fusion can be seen in the upper portion of fig. $3 \mathbf{A}$ and in the lower portion of fig. 3B. Ultimately, two microcysts were formed from this fusion pair, the upper two in fig. $3 \mathrm{I}$ fusing into one.

From a protuberance. When a broth culture is approximately $32-36 \mathrm{hr}$. old, the majority of organisms show an abrupt change in morphology. Transverse fission stops almost entirely and the organisms begin to shorten and become 
thicker (Pl. 2, fig. 4; Pl. 3, figs. 8, 9), many of them taking on a crescent shape. The crescent-shaped organism contains a single granule, which is observable in the living organism by both light and phase microscopes. It has been shown that this granule corresponds to the chromatin material of the fixed and stained organism (Williams, 1952). A protuberance may arise from the centre of the crescent-shaped organism which gradually enlarges and absorbs the remainder of the organism (Pl. 2, fig. 5).

Protuberances or swellings may also arise before the formation of the crescent-shaped forms and may occur at or near the ends as well as in the centre of the organisms (Pl. 3, fig. 6). Protuberances, produced at both ends of a single organism, may enlarge and merge into a single body (Pl. 3, fig. $6 \mathrm{E})$. A similar fusion of what appear to be almost fully formed microcysts is shown in Pl. 3, fig. 7. Fusion of microcysts also occurred in the contiguous organisms shown in Pl. 2, fig. 5, although, in this instance, the intermediate stages took place so rapidly that they were not photographed.

$B y$ the shortening of the entire organism. The majority of organisms neither fuse nor produce protuberances or swellings, but merely undergo a very gradual shortening and rounding, ending up as oval or spherical bodies (Pl. 3, figs. 8, 9).

The production of microcysts in any one culture of Spirillum lunatum is not synchronous, and freely motile organisms may be observed in cultures up to 3 weeks of age. For about a week longer, non-motile spiral organisms can still be seen, but after this time about the only formed material found in the cultures are the microcysts, which tend to settle to the bottom of the culture tube as a finely granular sediment (Pl. 4, fig. 10).

\section{Germination of microcysts}

When an old culture of Spirillum lunatum, completely in the microcyst stage, is transferred to new medium, no apparent change is observable by phasecontrast microscopy in the microcysts for the first few hours. When such material is fixed and stained, however, extensive changes in the chromatin material can be demonstrated. Eventually, at 6-8 hr. after inoculation, a germ tube emerges from the microcyst, which gradually lengthens until the normal spiral organism is produced. The time interval, from the first observance of the germ tube to the emergence of the complete spiral organism, varies from 3-6 hr. in microculture, but the germination time is considerably shorter in the normal broth culture. In the latter, the majority of microcysts have germinated by $16 \mathrm{hr}$.

Germination of the microcysts occurs by two methods; unipolar emergence of the germ tube (Pl. 4, fig. 11) and bipolar emergence (Pl. 4, fig. 12). The microcyst portion of the emerging vegetative organism is gradually absorbed and no coat is shed as is the case with some of the spore-forming bacilli and the Sporocytophaga.

\section{DISCUSSION}

The microcysts observed in Spirillum lunatum and other species of spirilla are apparently the same as the 'coccoid' bodies reported by earlier investigators (e.g. Ellis, 1903; Dimitroff, 1926), and similar bodies can be seen in 
photographs recently published by Cayton \& Preston (1955), who describe a new species of spirillum, $S$. mancuniense. With the exception of Dimitroff (1926), who compared the 'coccoid' bodies to those reported by Leishman (1918) for the spirochaetes of relapsing fever, either the microcysts were overlooked or else were dismissed as involution forms. However, from the data presented, it can be concluded that the microcysts represent a definite stage in a life cycle common to many spirilla.

The occurrence of life cycles in members of the Eubacteriales has been proposed from time to time (Lohnis, 1921; Hadley, 1937) but, with the exception of the spore-bacillus-spore cycle of the family Bacillaceae, is not generally accepted by bacteriologists. In the last 15 years, however, studies of L-forms by Klieneberger-Nobel (1949, 1951 $a, b)$, Dienes \& Weinberger (1951), Tulasne (1953), and others have shown that various Eubacteriales may reproduce by methods other than transverse fission.

The fusion forms observed in 20-30 hr. cultures of Spirillum lunatum resemble those shown by Klieneberger-Nobel for the Morax-Axenfeld bacillus (her figures 1 to $12 ; 1951 a$ ). The protuberances produced by organisms of $S$. lunatum are similar to those shown by Stempen (1955) for Proteus vulgaris (his figures $\mathbf{2}$ and 3). Fusion of organisms has also been shown by Hutchinson \& Stempen (1954), but these authors doubted whether this process is a sexual one. Plasmogamy without caryogamy is common in the fungi and the fusion observed with the spirilla does not necessarily represent a sexual fusion, although evidence from fixed and stained preparations (to be presented later) suggests that the fusion of the organisms is followed by nuclear fusion.

The similarity in appearance, mode of formation, and mode of germination between the microcysts of Spirillum lunatum and those of the Sporocytophaga, together with the fact that the latter organisms have been described, in the past, as having a spiral form, might lead one to suspect that $S$. lunatum is merely a mis-identified Sporocytophaga. The presence of polar flagella and a very active swimming motility rules out this possibility (Williams, 1952). More important, the similarities mentioned above suggest that the eubacteria and the myxobacteria may not be as divergent, phylogenetically, as some authorities believe.

\section{REFERENCES}

Cayton, H. R. \& Preston, N. W. (1955). Spirillum mancuniense n.sp. J. gen. Microbiol. 12, 519.

Dienes, L. \& Weinberger, H. J. (1951). The L forms of bacteria. Bact. Rev. 15, 245.

Dimitroff, V. T. (1926). Spirillum virginianum nov. spec. J. Bact. 12, 19.

Ellis, D. (1903). Untersuchungen über Sarcina, Streptococcus und Spirillum. Z Zbl. Bakt. (Abt. 1), 33, 81.

Grace, J. B. (1951). The life cycle of Sporocytophaga. J.gen. Microbiol. 5, 519.

Hadley, P. (1937). Further advances in the study of microbic dissociation. $J$. infect. Dis. 60, 129.

Hutchinson, W. G. \& Stempen, H. (1954). Sex in bacteria. Evidence from morphology. In Sex in Microorganisms. Washington, D.C.: American Association for the Advancement of Science. 
Journal of General Microbiology, Vol. 15, No. 1

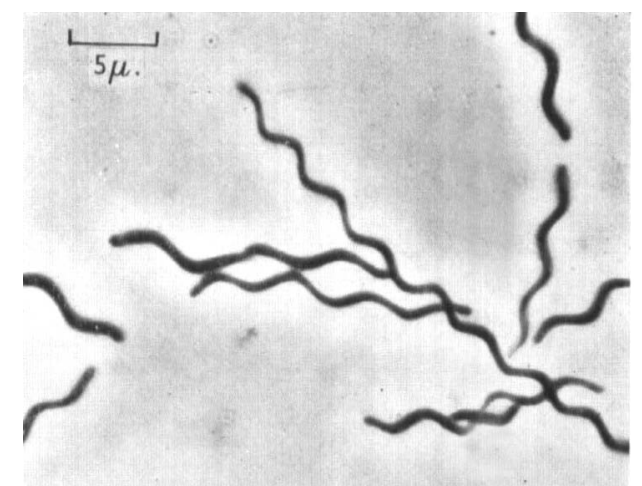

Fig. 1.

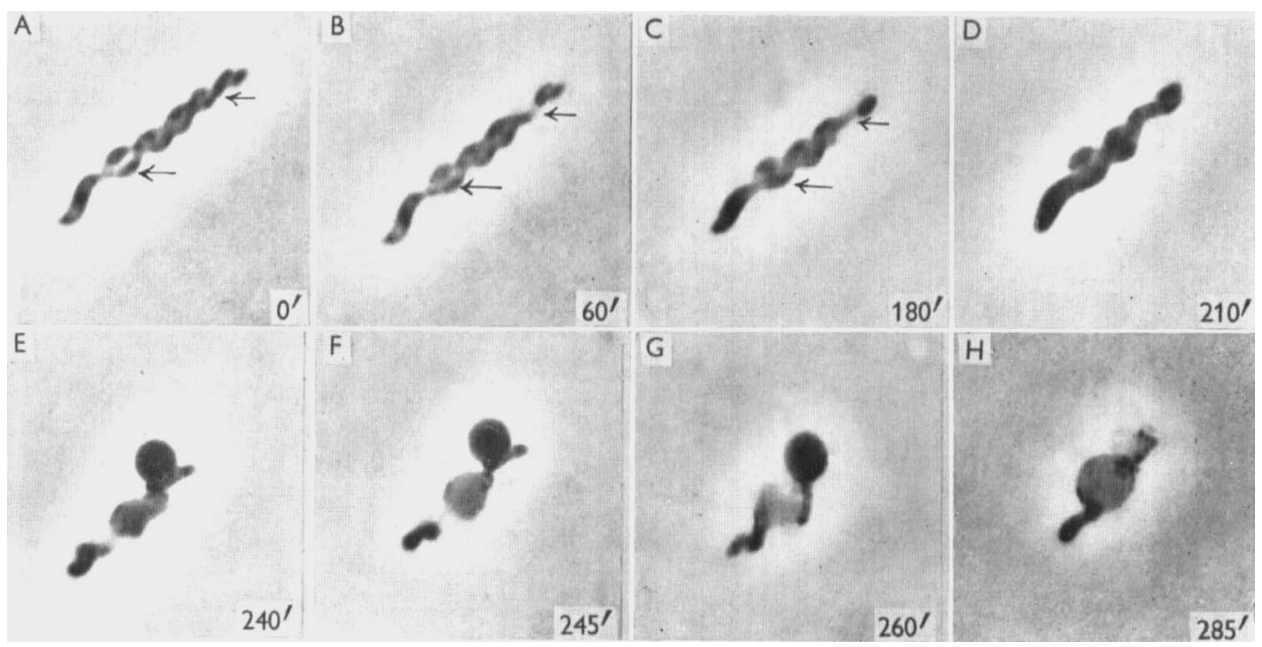

Fig. 2.

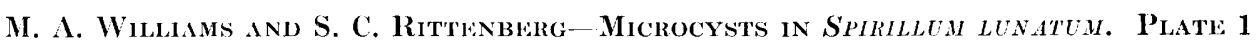


Journal of General Microbiology, Vol. 15, No. 1

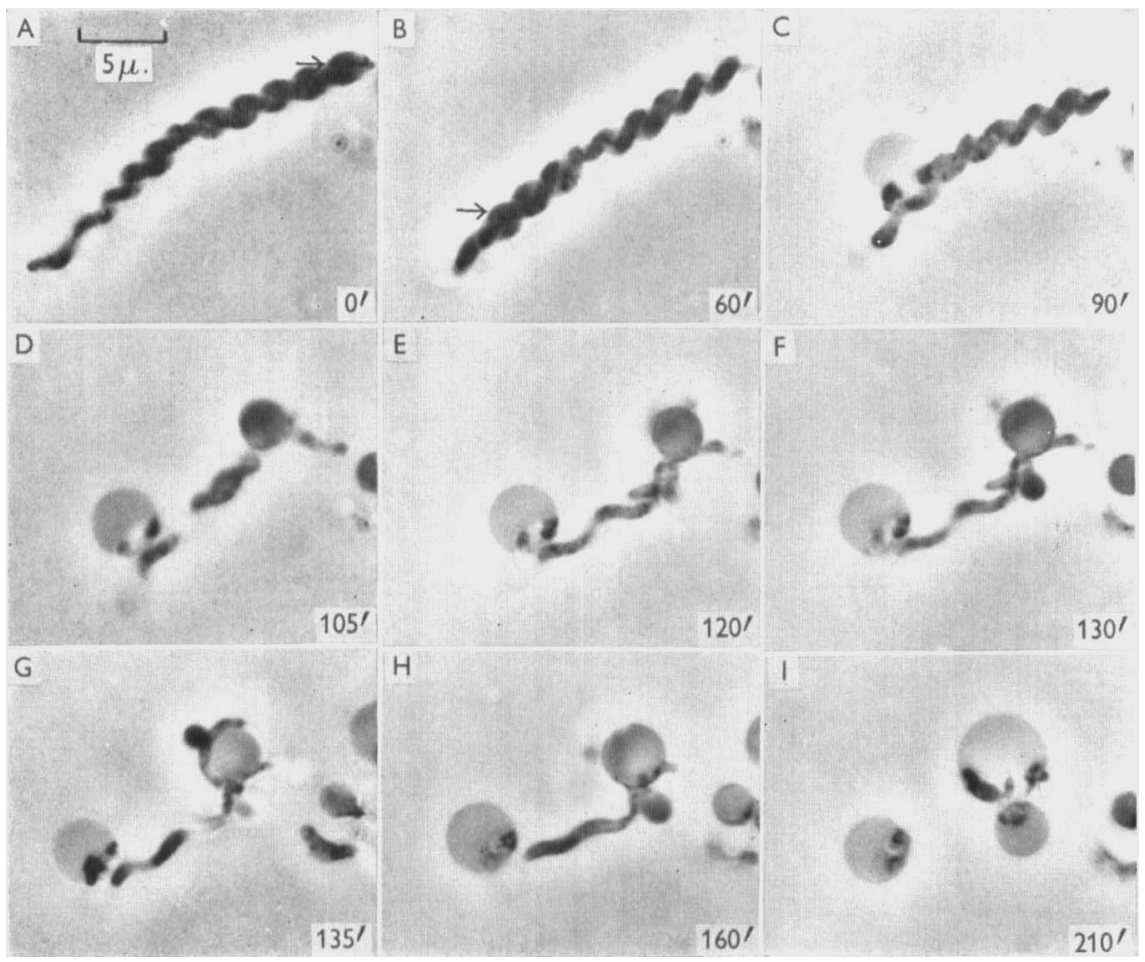

Fig. 3.

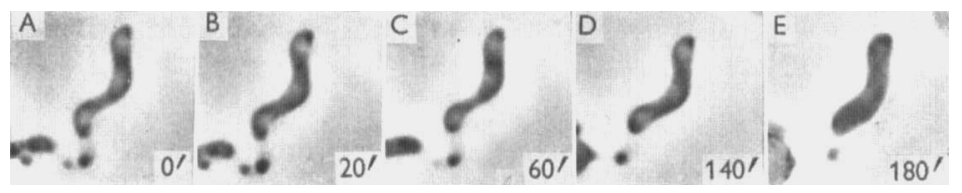

Fig. 4.

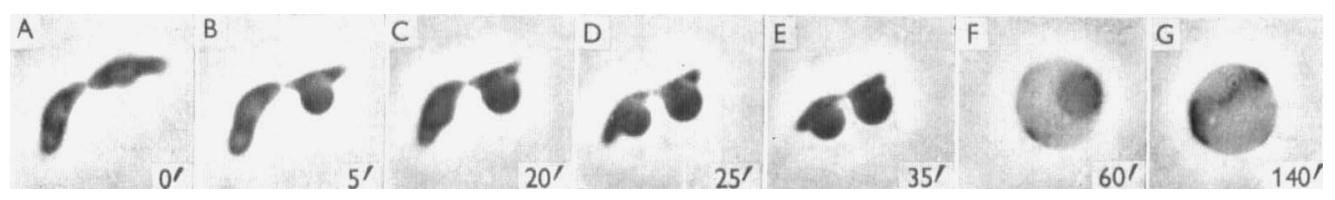

Fig. 5.

M. A. Williams and S. C. Rittenberg-Microcysts in Spikillum lunatum. Plate 2 
Journal of General Microbiology, Vol. 15, No. 1
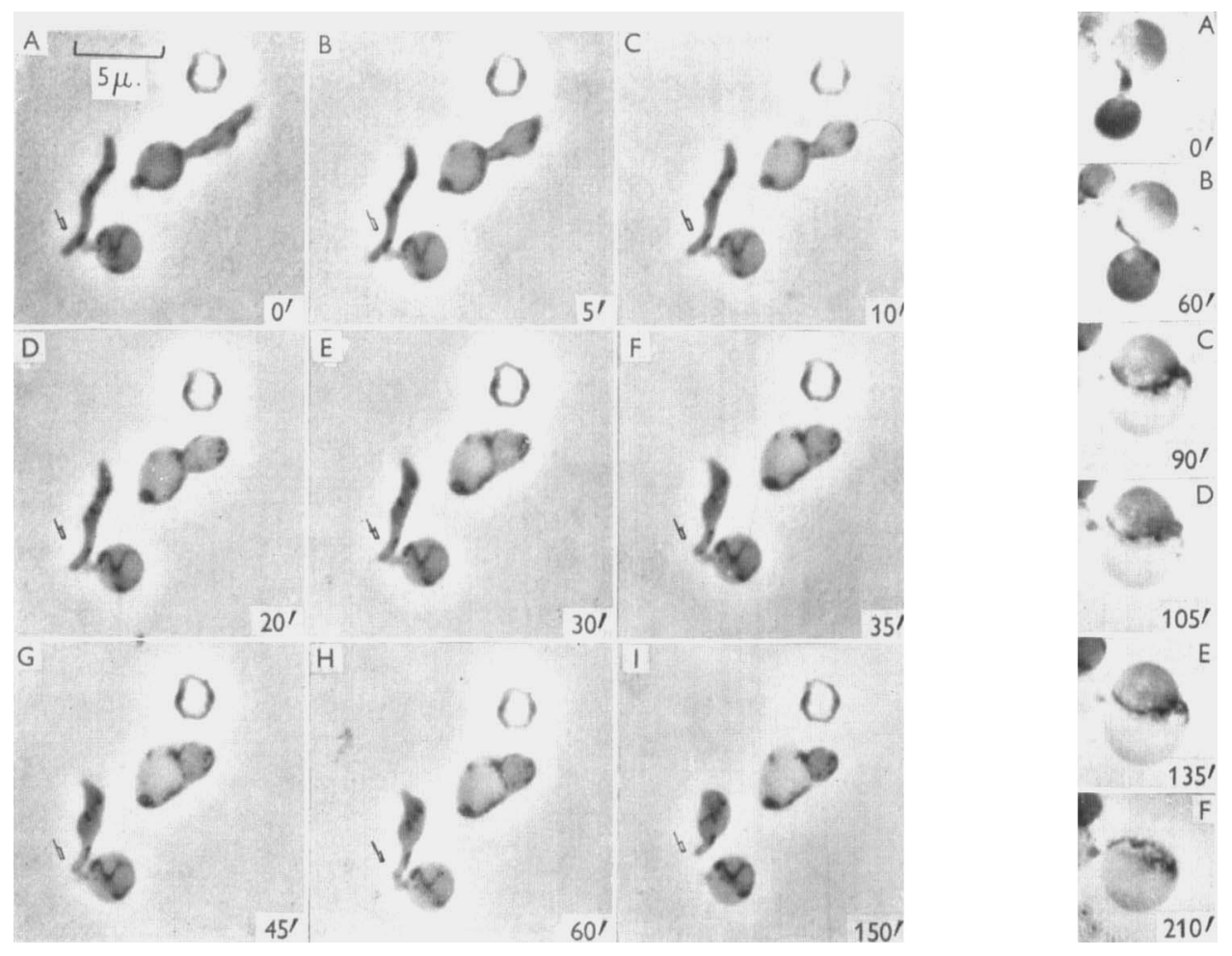

Fig. 6.

Fig. 7.

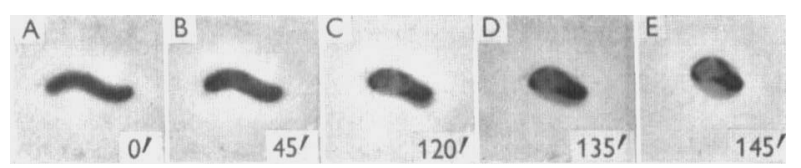

Fig. 8.

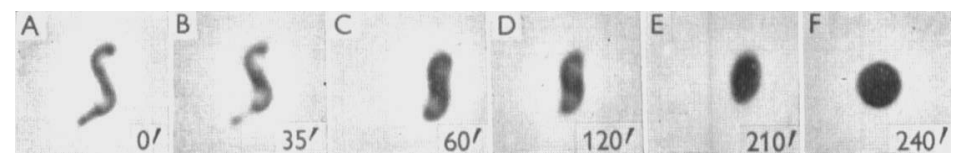

Fig. 9.

M. A. Williams and S. C. Ritilenberg-Michocysts in Spirillem lunatum. Plate 3 
Journal of General Microbiology, Vol. 15, No. 1

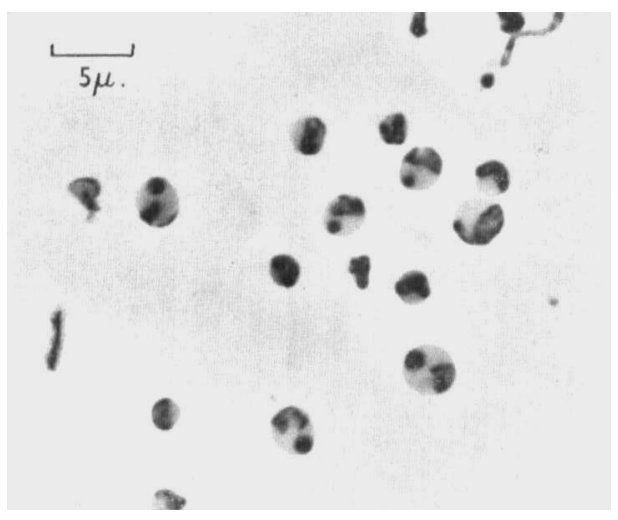

lïg. 10.

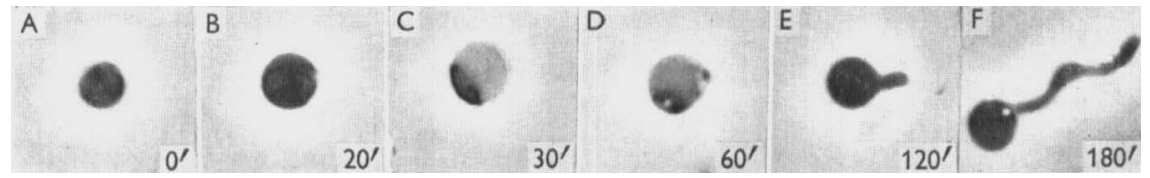

I'ij. 11 .

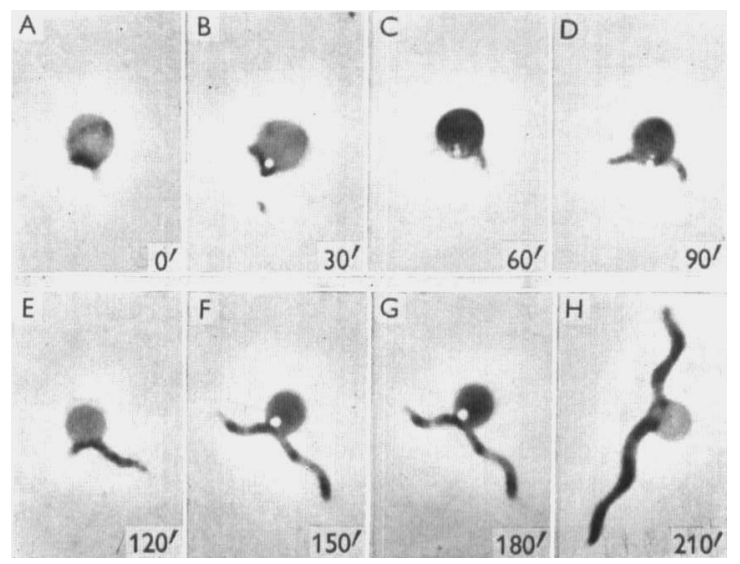

Fig. 12.

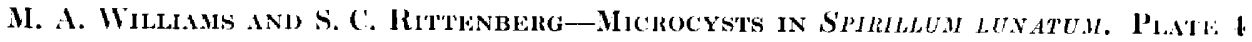




\section{Microcysts in Spirillum lunatum}

Klieneberger-Nobel, E. (1949). Origin, development, and significance of L-forms in bacterial cultures. J. gen. Microbiol. 3, 434.

KLIENEBerger-Nobel, E. $(1951 a)$. The L-cycle: a process of regeneration in bacteria. J. gen. Microbiol. 5, 525.

Klieneberger-Nobel, E. (1951 b). Filterable forms of bacteria. Bact. Rev. 15, 77.

Leishman, W. B. (1918). A note on the 'Granule Clumps' found in Ornithodorus moubata and their relation to the spirochetes of relapsing fever (tick fever). Ann. Inst. Pasteur, 32, 49.

Lohnis, F. (1921). Studies upon the life cycles of bacteria. Memoirs Nat. Acad. Sciences (Washington, D. C.), 2nd memoir, 16, 1.

Stanier, R. Y. (1942). The Cytophaga group: a contribution to the biology of Myxobacteria. Bact. Rev. 6, 143.

Stempen, H. (1955). Demonstration of a cell wall in large bodies of Proteus vulgaris. J. Bact. 70, 177.

Tulasne, R. (1953). Le cycle $L$ et les formes naines des bactéries. Symposium: Bacterial Cytology. Springfield, Illinois: Charles C. Thomas.

Wrluiams, M. A. (1952). A Study of the Genus Spirillum Ehrenberg. M.A. Thesis, University of Southern California, Los Angeles.

\section{EXPLANATION OF PLATES}

\section{Plate 1}

All photographs are of living Spirillum lunatum made by phase-contrast microscopy. The scale of fig. 1 holds for all photographs.

Fig. 1. Normal vegetative organisms of $S$. lunatum. $26 \mathrm{hr}$. broth culture.

Fig. 2. Microcyst formation following fusion of organisms. The arrows show the points of fusion. Organisms from a $24 \mathrm{hr}$. broth culture.

\section{Plate 2}

Fig. 3. Microcyst formation following fusion. Arrows show upper and lower points of fusion. Organisms from a $26 \mathrm{hr}$. broth culture.

Fig. 4. Shortening of organisms with production of crescent-shaped form prior to microcyst formation. Organisms from a $30 \mathrm{hr}$. broth culture.

Fig. 5. Microcyst formation from a protuberance from the middle of a crescent-shaped form. The microcysts from the contiguous organisms have fused. Organisms from a $30 \mathrm{hr}$. broth culture.

\section{Plate 3}

Fig. 6. Fusion of microcysts formed from protuberances at opposite ends of organism. Organism ' $b$ ' also shows protuberance formation and gradual shortening into a microcyst. Organisms from a $36 \mathrm{hr}$. broth culture.

Fig. 7. Fusion of two spherical bodies into a single microcyst. Organisms from a $24 \mathrm{hr}$. broth culture.

Fig. 8. Microcyst formation by shortening and rounding. Organism from a $36 \mathrm{hr}$. broth culture.

Fig. 9. Microcyst formation by shortening and rounding. Organism from a $24 \mathrm{hr}$. broth culture.

\section{Plate 4}

Fig. 10. Microcysts from a 4-week old culture.

Fig. 11. Unipolar germination of microcyst. Microcysts transferred from a 4-week old culture into fresh medium; sequence started after $10 \mathrm{hr}$. in new culture.

Fig. 12. Bipolar germination of microcyst. Microcysts transferred from a 4-week old culture into fresh medium; sequence started after $12 \mathrm{hr}$. in new culture.

(Received 23 February 1956) 